\title{
Use of Randomized Submaximal Glutamate Stimulus to Interpret Glial Effects on Neuronal Calcium Dynamics
}

\author{
Kinsey C Kelly ${ }^{1}$, Katie Evans ${ }^{2}$, Mihaela Paun ${ }^{3}$ and Mark A DeCoster ${ }^{4,5 *}$ \\ ${ }^{1}$ Department of Biophysics, Centenary College, Shreveport, LA, USA \\ ${ }^{2}$ Department of Mathematics and Statistics, Louisiana Tech University, Ruston, LA, USA \\ ${ }^{3}$ Bioinformatics Department, National Institute of Research and Development for Biological Sciences, Bucharest, Romania \\ ${ }^{4}$ Department of Biomedical Engineering, Louisiana Tech University, Ruston, LA, USA \\ ${ }^{5}$ Institute for Micro Manufacturing, Louisiana Tech University, Ruston, LA, USA
}

\begin{abstract}
Glutamate (GLU) binding to neurons can cause dynamic changes in intracellular calcium. We tested effects of a 3-group submaximal glutamate stimulus (250,500 and 750 nanomolar GLU in randomized orders) on neurons in culture, and measured intracellular calcium dynamics in cultures high and low in glia at 8 and 9 days in vitro. Gliadepleted cultures responded to increasing GLU with synchronized dynamics, leading to a greater "area under the curve" (AUC) for intracellular calcium over time. The AUC determined if the neuron would respond dynamically to the next addition of glutamate. This observation was not displayed within cultures high in glia, where AUC returned to baseline with every GLU addition, regardless of order of addition. Furthermore, the 3-group stimulus resulted in decreasing average AUC, regardless of order. In contrast, for cultures depleted of glia, the deciding factor of a responding cell to dynamically respond to GLU additions depended on the ability of the cell to distribute the calcium load (AUC) of the prior addition. Determining how neurons respond and behave such as in the presence of functional or dysfunctional glia, may help our understanding of signal processing in the brain.
\end{abstract}

Keywords: Glutamate; Calcium; Neurons; Astrocytes

\section{Introduction}

The neuronal synapse is a dynamic functional unit which is composed of neurons and support glial cells, which provide the important function of glutamate re-uptake [1]. We have previously described calcium dynamics in neuronal cultures treated with toxic and non-toxic concentrations of glutamate using single stimuli of micromolar glutamate [2]. Here we investigated 1) whether a pattern of multiple, submaximal glutamate stimulations at nanomolar concentrations of glutamate affected calcium dynamics in neuronal cultures with and without high glial content, and 2) whether the order of addition of nanomolar glutamate affected calcium dynamics. Calcium is a key signaling ion involved in memory and learning with ionotropic glutamate receptors such as the $\mathrm{N}$-methyl-D-aspartate receptors (NMDAR) on the neuronal membrane [3]. NMDARs are a major subtype of ionotropic receptors responsible for binding glutamate, the most abundant excitatory neurotransmitter (excitatory stimulus) in the human brain. Activation of NMDARs opens the ion channel at the plasma membrane to allow calcium influx into the cell cytosol. Calcium in its ionic form is very dynamic, especially in excitable cells such as muscle and brain cells, moving from the high concentration exterior of the cell to the much lower concentrations inside the cell where calcium is used as a second messenger. In brain cells and neurons especially, calcium is a key signaling ion involved in memory and learning with excitatory neurotransmitters such as glutamate turning neurons "on". Glutamate excites the neurons in part by causing large and dynamic changes in intracellular calcium concentration $\left(\left[\mathrm{Ca}^{2+}\right]_{\mathrm{i}}\right)$ increases. While these $\left[\mathrm{Ca}^{2+}\right]_{i}$ dynamics are essential for normal signaling in the brain, excessive and sustained elevations in neuronal $\left[\mathrm{Ca}^{2+}\right]_{i}$ are related to neuronal injury [4] including long-term neurodegenerative processes [5]. Helping to regulate these dynamics in the brain are the glial cells known as astrocytes. Astrocytes express glutamate transporters [6], and in this way diminish the time that neurons are exposed to glutamate, and thus also shaping the $\left[\mathrm{Ca}^{2+}\right]_{i}$ dynamics in neurons, and in general, decreasing neuronal $\left[\mathrm{Ca}^{2+}\right]_{\mathrm{i}}$ increases for a given concentration of glutamate by removing it from the synapse. NMDARs can be excessively stimulated by glutamate, which can lead to an abundance of calcium influx into the cytosol which will cause neuronal damage and can lead to cell death, termed excitotoxicity [7]. Glial cells are responsible for removing excess glutamate from the surrounding environment, but are limited to the amount of glutamate they can take up. Glutamate homeostasis is maintained by neuronal release, neurotransmission, and glial uptake. When exogenous glutamate is introduced to cells in vitro (as we have done here), at some threshold we expect calcium to enter the neurons, because glutamate is an excitatory neurotransmitter. Furthermore, the more one stimulates neurons with glutamate (the higher the glutamate concentration), the higher the possibility of some calcium influx. In the experimental realm shown here, calcium influx into the cells is expressed as an increase in fluorescence, due to the fluorescent probe for calcium that we are using, Fluo-3/AM [2]. Here we tested whether the order of stimulus using a 3-group concentration $(250,500$, and $750 \mathrm{nM})$ of glutamate could be used to predict neuronal responses in cultures either high or low in glial content. Here we describe an in vitro cell culture system composed of rat brain cortical neurons with different densities of astrocytes which we have used to analyze the $\left[\mathrm{Ca}^{2+}\right]_{\mathrm{i}}$ dynamics in individual neurons. This work follows our long-standing interest in brain cell $\left[\mathrm{Ca}^{2+}\right]_{\mathrm{i}}$ dynamics [2], but with the applied randomization

*Corresponding author: Mark A DeCoster, Biomedical Engineering, Louisiana Tech University, PO Box 10157, Ruston, Louisiana, LA 71270, USA, Tel: 318-2575233; Fax: 318-257-4000; E-mail: decoster@latech.edu

Received November 23, 2015; Accepted January 04, 2016; Published January 08, 2016

Citation: Kelly KC, Evans K, Paun M, DeCoster MA (2016) Use of Randomized Submaximal Glutamate Stimulus to Interpret Glial Effects on Neuronal Calcium Dynamics. Neurochem Neuropharm Open Access 1: 107. doi:10.4172/24699780.1000107

Copyright: ( 2016 Kelly KC, et al. This is an open-access article distributed under the terms of the Creative Commons Attribution License, which permits unrestricted use, distribution, and reproduction in any medium, provided the original author and source are credited. 
of stimulus order we now investigate here a system for predicting: 1) whether the order of repeated glutamate stimulation alters neuronal $\left[\mathrm{Ca}^{2+}\right]_{\mathrm{i}}$ dynamics and 2) how the presence of different densities of astrocytes modulates neuronal $\left[\mathrm{Ca}^{2+}\right]_{\mathrm{i}}$ dynamics. We anticipate that this combined experimental/analytical approach will also have utility in understanding additional brain diseases such as brain tumors [8].

\section{Materials and Methods}

\section{Primary cortical culture preparation}

Cortical cells were obtained by performing cervical disarticulation of Outbred Sprague-Dawley newborn rats (age $\leq 48 \mathrm{hrs}$ ) in adherence to protocols approved by Louisiana Tech University's Ethics and Animal Care Committee and as detailed previously [9]. Rats were decapitated and the brain tissue was quickly removed and placed into dissecting solution, Basal Media Eagle (BME, Sigma) with $0.5 \%$ Penicillin/Streptomycin (PS). The cerebellum and meninges were removed, and the lobes were then contained in ice-cold dissecting solution. An average of $n=7$ newborn rats were used for each culture set. After dissection was completed, the brain tissue was then aspirated with a $5 \mathrm{~mL}$ pipette and placed into a $15 \mathrm{~mL}$ conical tube with a complementing volume of Trypsin EDTA (Sigma) and inverted 5 times. Trypsin was then neutralized with Neuronal Culture Medium (NCM) comprised of BME, Ham's F-12 K (ATCC), 10\% Horse Serum, $10 \%$ Fetal Bovine Serum, and supplemented with glucose, glutamine and PS as previously described [9]. The cells were then mechanically disassociated by trituration and allowed ten minutes to form a neuronal cell supernatant. The supernatant was then aspirated and stored in a $15 \mathrm{~mL}$ ice-cold conical tube. NCM was then re-introduced to the brain tissue and the process repeated thrice in total. The neuronal cell supernatant was centrifuged at $160 \times \mathrm{g}$ at $8^{\circ} \mathrm{C}$ for 7 minutes to form a pellet. Once the cells were resuspended in fresh NCM, a cell count was obtained with a hemacytometer; the cells were then plated into a 24 multi-well plate (cell culture treated, Cellstar) at an optimal density of 200,000 cells per well. The cell cultures were maintained in a $37^{\circ} \mathrm{C}$, $5 \% \mathrm{CO}_{2}$ humidified incubator. After three days in vitro, the cell culture plates were split in half, with one half of the culture treated with a $100 \mathrm{x}$ dilution of Cytosine Arabinoside ([Ara C] $1 \mathrm{mM}$, Sigma) to deplete glial cells from cultures. The remaining wells were supplemented with warmed NCM and were used for co-culture experiments.

\section{Calcium fluorescence imaging}

The cortical cultures were imaged after 8 to 9 days in vitro, by incubating cells in a loading solution of the calcium indicator Fluo3 AM (Invitrogen), as previously described [10]. The cells were then washed and recovered in warmed Locke's solution and re-incubated for 30 minutes, while the cells were recovering, fresh Glutamic acid ((GLU) 250, 500 and $750 \mathrm{nM}$, Sigma Aldrich) concentrations were prepared in Locke's solution. The cells were imaged with an Olympus CKX41 inverted microscope with a 488 excitation wavelength filter over real time at a $4 \mathrm{~s}$ frame rate with Intracellular Imaging software. A baseline (recording of cells without treatment) was obtained for $60 \mathrm{~s}$, and GLU concentrations were added to the experiment at predetermined intervals $(60,320$, and $580 \mathrm{~s})$ without washing out the media between additions.

\section{Measurement and analysis of fluorescence intensity}

Intracellular imaging software (InCyt Im Imaging system, version $5.29 \mathrm{e}$, Cincinnati, OH) was utilized to create regions of interest (ROIs) around every cell in the data set post experiment, for all cells showing at least a 1.2-fold (20\%) increase in fluorescence compared to starting (time 0) values. ROIs were generated around neurons and not astrocytes on the basis of cell morphology and responses to glutamate at these very low glutamate concentrations (nM). In separate experiments, astrocytes in the cultures were identified on the basis of immunocytochemical staining with glial fibrillary acidic protein (GFAP) and $\left[\mathrm{Ca}^{2+}\right]_{\mathrm{i}}$ responses to direct glutamate stimulation (data not shown). We found that astrocytes demonstrated $\left[\mathrm{Ca}^{2+}\right]_{\mathrm{i}}$ responses to glutamate consistently only in the high micromolar and low millimolar range, which is consistent with a number of previous reports [11-13] The ROIs were then used to measure fluorescence intensity over time in the specified area and created a data set based on ROIs. At least three different platings from at least three different primary culture preparations were used for every condition and combination of glutamate stimuli reported in this work. A total of 773 cells (regions of interest) were included in analysis in the various figures shown.

\section{Calculation of area under the curve (AUC)}

Analyses were used to determine the Area Under the Curve (Figure 2) of a cell's intracellular calcium response to GLU. This parameter was calculated for the fluorescence-intensity time curve, $\mathrm{f}$, for each cell as follows:

$$
\int_{a}^{b} f(x) d x \approx(\mathrm{b}-\mathrm{a}) \frac{f(a)+f(b)}{2}
$$

Where times $a$ and $b$, and their corresponding fluorescence intensities are $\mathrm{f}(\mathrm{a})$ and $\mathrm{f}(\mathrm{b})$ at the time interval's endpoints. A summation of evaluated areas is then compiled for every ROI. The data utilized was normalized to the starting (time 0 ) value for each ROI, by dividing all values for that ROI by its time 0 value; thus all ROI values start at 1 .

\section{Results}

The glial content of neuronal cultures was controlled by treatment with cytosine arabinoside (AraC) as previously described [10]. Figure 1 shows schematics and merged images of typical cultures with low and high glial content and associated calcium responses after glutamate stimulation. These static images indicate that AraC-treated cultures
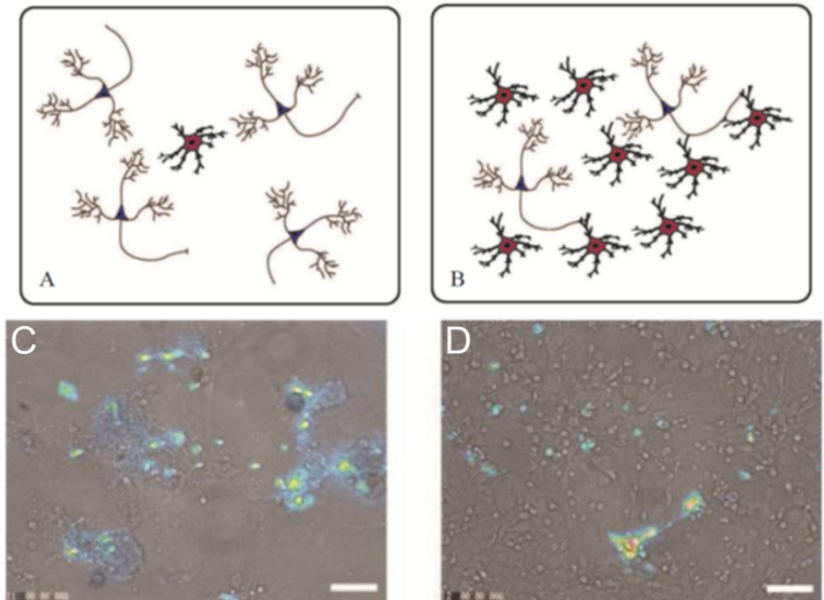

Figure 1: Neuronal cultures with low and high glial content: schematic representations $(A+B)$ and merged phase and pseudocolor images $(C+D)$ are shown. A) Primary cortical cultures after treatment with AraC to deplete cultures of glia. B) Primary cortical pictures high in glia. Triangular bodies=neurons, Round bodies=glia. C) Phase image merged with calcium fluorescence of primary cortical culture treated with AraC. D) Phase image of primary cortical cultures high in glia with merged calcium fluorescence image. Scale bar=50 microns. 


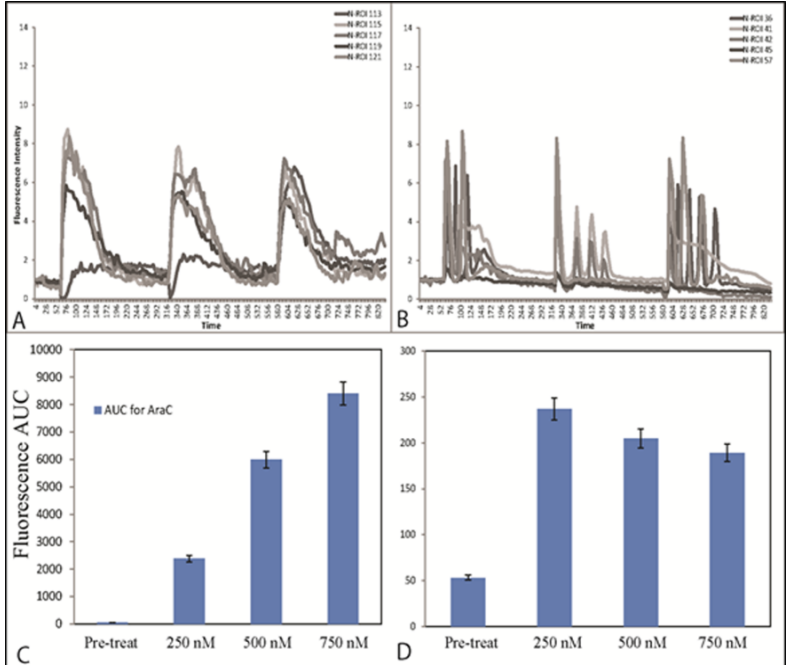

Figure 2: Increasing concentrations of glutamate represented as line tracings and area under the curve (AUC). A) Line representations of increasing glutamate in cultures low in glia. B) Line representations of increasing glutamate in cultures high in glia. C) Bar graph of AUC for increasing glutamate in cultures low in glia. D) Bar graph of AUC for increasing glutamate in cultures high in glia. A+B: 5 example neurons are shown. $C+D$ : average of all neurons for each set shown, with error bars indicating S.E.M. $\mathrm{N}=127$ neurons for panel $2 \mathrm{C} ; \mathrm{N}=116$ neurons for panel 2D. Pre-treat indicates measured AUC during baseline period before the first GLU stimulus of $250 \mathrm{nM}$.

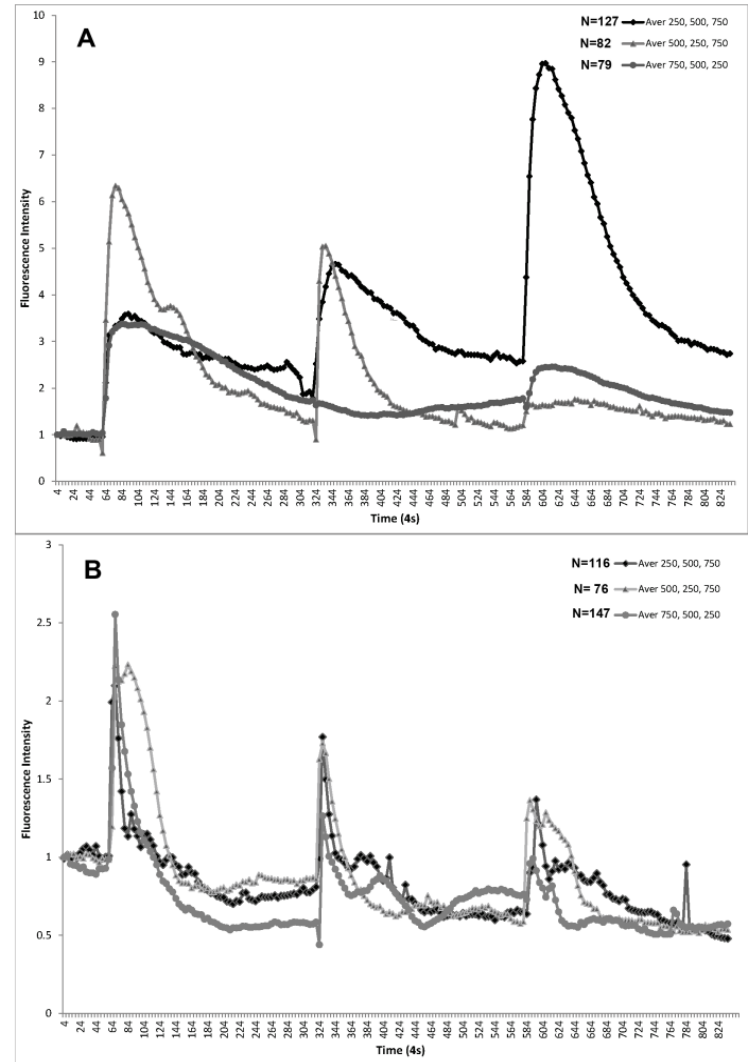

Figure 3: Comparison of randomized stimulus per culture environment A) Averaged Line tracings in low glia environment in response to multiple randomized glutamate stimuli. B) Averaged Line tracings in high glia environment in response to multiple randomized glutamate stimuli. Stimulation order for each culture condition is indicated by symbol; $\mathrm{N}=\#$ of cells analyzed for each condition. demonstrate a network of individual neurons and groups of neurons connected by long processes (Figure 1A and 1C). In contrast, cultures without AraC (high glial content) demonstrate a full monolayer of cells including neurons and glia, with neurons showing clear responses to nanomolar glutamate stimulation (Figure 1B and 1D). We next wanted to determine whether multiple subthreshold stimuli with glutamate (nanomolar concentrations) would affect neuronal cultures with and without AraC differently. The same sequence of three stimuli, that is, 250,500 , and $750 \mathrm{nM}$ glutamate, was used on neuronal cultures with and without $\mathrm{AraC}$, with equal time spacing between each stimulation. Glutamate, once added to the cultures, was not washed out, therefore taking into account glutamate reuptake as a way for the cultures to return to baseline calcium levels. As expected, cultures treated with AraC (low glial content), showed successively broader peaks of calcium increases as indicated by area under the curve (AUC) analysis (Figure 2A and 2C). In contrast, cultures without AraC (high glial content) demonstrated more spikey calcium dynamics in response to these same glutamate stimuli (Figure 2B), with AUC analysis showing response many-fold less than cultures with higher neuronal content (Figure 2D). It is important to note that in all cases, the three successive stimuli for all cultures were considered sub-threshold in that cells returned spontaneously towards baseline levels of calcium, without washing out the added glutamate. We then directly compared these dynamic tracings for averages of many neurons for three different orders of glutamate addition for cultures depleted of glia (Figure 3A), or high in glia (Figure 3B). It can be clearly seen that the amplitude of response, and total area under the curve for neurons treated with glutamate, is much higher in cultures depleted of glia (Figure 3A) compared to cultures high in glia (Figure 3B), as would be expected from excitotoxicity studies using glutamate [14]. When glutamate is used to stimulate cells in the order of 250,500 , and $750 \mathrm{nM}$ glutamate, as expected, this increasing concentration of successive glutamate stimuli results in increasing amplitude of response and increasing AUC (Figure 3A, dark line, diamond symbols). Altering the order of glutamate stimulus to 500, 250, and $750 \mathrm{~nm}$ GLU results in two robust neuronal responses followed by a shallow but broad peak in response to the final stimulus (Figure 3A, triangle symbols). Finally, stimulation in the order of 750,500 , and $250 \mathrm{nM}$ GLU results in a large, broad AUC in response to the first stimulus, with no response on average to the second stimulus, and a final robust reponse to $250 \mathrm{nM} \mathrm{GLU}$ which remains above the starting baseline (Figure 3A, circle symbols). In contrast to this, on average, for all neurons in cultures high in glia, the following is observed: 1) lower amplitude and smaller AUC is observed for all orders of GLU stimulus (Figure 3B) compared to cultures low in glia (Figure 3A and 3B) the amplitude and AUC of each successive addition of GLU in cultures high in glia decreases, from $1^{\text {st }}$ to $3^{\text {rd }}$ stimulus, regardless of the order or GLU concentration (Figure 3B). This is in stark contrast to cultures low in glia (Figure 3A). Thus, in all stimulation cases ( 9 of 9), the average peak height (amplitude) decreased with successive glutamate treatments (stimulus order did not matter: Figure 3B) while for low-glial content cultures, the stimulus order did matter in regard to stimulus response amplitude (Figure 3A). Four different GLU stimulation protocols were used in the experiments described here and are shown for pairwise comparison in cultures low and high in glial content in Figure 4A-4D. Using the same fluorescent intensity axis for direct comparison of cultures low and high in glial content we can again see expected stepwise increases in amplitude and AUC as we increase GLU stimulus from 250-750 nM (Figure 4A). Starting with the two highest GLU stimuli (500 and $750 \mathrm{nM}$ ) followed by $250 \mathrm{nM}$ also results in stepwise increases in amplitude and AUC in cultures depleted of glia (Figure 4B). Stimulation with 500 nM GLU 

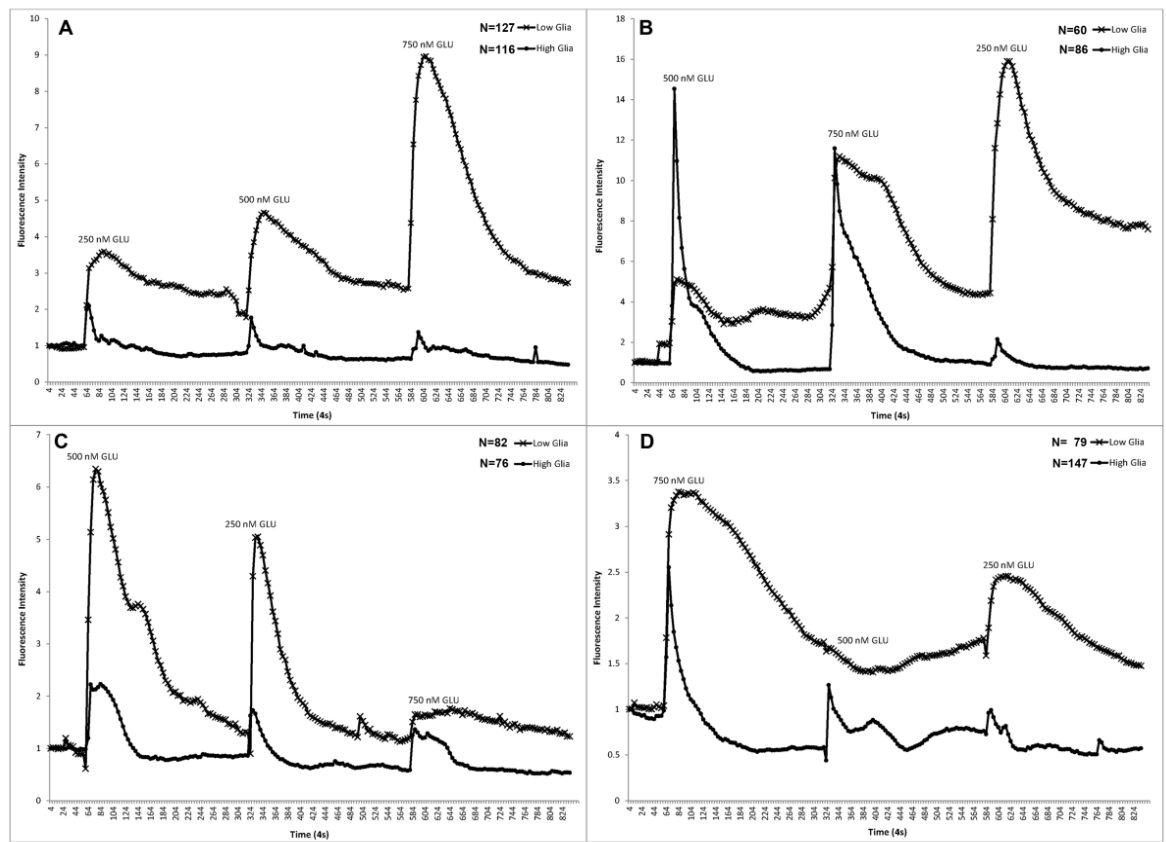

Figure 4: Pairwise comparison of GLU responses in low glia vs. high glia cultures. A) non-randomized (increasing concentration) additions of GLU. B) randomization of glutamate stimulus: $500,750,250 \mathrm{nM}$. C) randomization of glutamate stimulus: $500,250,750 \mathrm{nM}$. D) randomization of glutamate stimulus: $750,500,250$ nM. N=\# of cells analyzed for each condition. All line tracings represent averaged response for the data set for the given condition.

followed by 250 results in two robust but transient peaks, and a final low, but broad peak in response to the final addition of $750 \mathrm{nM}$ GLU (Figure 4C). When naïve cells were treated at the outset with $750 \mathrm{nM}$ GLU, the response was robust and broad, with amplitude remaining above baseline before the second addition of $500 \mathrm{nM}$ GLU, which showed no response, until time had passed when $250 \mathrm{nM}$ GLU was added and cells responded again (Figure 4D). For every single one of these permutations in the ordering of GLU concentration stimulations, cultures high in glia demonstrated lower amplitude responses and smaller AUC over time from $1^{\text {st }}$ addition through $3^{\text {rd }}$ addition regardless of the GLU concentration used. (Figure 4A-4D). In almost all cases (10 of 12 combinations), the average peak heights (or amplitudes) for responses in low-glial content cultures were higher than for high-glial content cultures. Furthermore, in all cases (12 of 12 combinations), the AUC was higher for each stimulus and for each stimulus combination in the low-glial content cultures (Figure 4).

\section{Discussion}

We have made novel observations of the ability of submaximal concentrations of GLU (nanomolar) to elicit intracellular calcium concentration dynamics in neurons in cultures low or high in glial content. As expected, cultures low in glia on average demonstrated higher amplitude neuronal $\left[\mathrm{Ca}^{2+}\right]_{\mathrm{i}}$ response to GLU addition since it is well known that glia (astrocytes) take up glutamate [6] and cultures low in glia are injured to a greater extent by glutamate than cultures high in glia [14]. We had previously shown that for a single glutamate stimulus in the micromolar range, that increasing concentrations of glutamate resulted in greater AUC as expressed as a slower (or lack of) recovery to baseline $\left[\mathrm{Ca}^{2+}\right]_{\mathrm{i}}[2]$. Here, we chose the novel approach of treating with 3 concentrations of glutamate all in the nanomolar range, and added to the cells in different random orders. Furthermore, we did not wash out the GLU once added to the cultures, so we are confident that the return to baseline $\left[\mathrm{Ca}^{2+}\right]_{i}$ is a good indicator of intrinsic capabilities of the culture to take up glutamate, for example by astrocytes [15]. In this regard, it is therefore not surprising that cultures high in glia showed much more transient responses in $\left[\mathrm{Ca}^{2+}\right]_{i}$ when stimulated with glutamate compared to cultures low in glia. From our results it appears that a good predictor of neuronal response to GLU stimuli in this submaximal range $(250-750 \mathrm{nM})$, is the AUC and return to baseline $\left[\mathrm{Ca}^{2+}\right]_{i}$ levels of the cells to the prior GLU stimulus. Thus, in the case of naive cells stimulated with the highest GLU concentration $(750 \mathrm{nM})$, a robust response and broad peak not returning to baseline, results in desensitization to the second stimulus of $500 \mathrm{nM}$, but given sufficient time, the cells do respond to the final stimulus of $250 \mathrm{nM}$ GLU (Figure 3A). In contrast, on average, all cells in cultures high in glia showed lower amplitude and smaller AUC for $\left[\mathrm{Ca}^{2+}\right]_{\mathrm{i}}$ regardless of the stimulation order (Figure 3B). When directly comparing cultures that are low or high in glia, we see a general trend of cells in culture deprived of glia increasing in $\left[\mathrm{Ca}^{2+}\right]_{i}$ as we increase GLU concentration, and decreasing as we decrease GLU (Figure 4A and 4B). However, we propose that a good predictor of neuronal response to a second or third submaximal GLU stimulus may be whether the cells have fully returned towards baseline levels of $\left[\mathrm{Ca}^{2+}\right]_{i}$ before the next stimulus. For example, as shown in Figure 4D, cells treated initially with 750 nM GLU have not fully recovered before addition of $500 \mathrm{nM}$ GLU, and thus no response on average is observed, until sufficient time has passed and then a cell response is seen to $250 \mathrm{nM}$ GLU. Remarkably, for all of these pairings we observed that in cultures high in glia, the $\left[\mathrm{Ca}^{2+}\right]_{\mathrm{i}}$ amplitude and AUC on average always decreased from $1^{\text {st }}$ to $3^{\text {rd }}$ stimulus, regardless of the order or concentration of GLU stimulation using the same combinations and concentrations as were used for cultures low in glia. To our knowledge this has not been observed before, and this may reflect the ability of neurons at synapses densely surrounded by astrocytes to more effectively avoid desensitization compared to those with low glial content [16]. One possible explanation for our results is that as we increase effective glutamate concentration 
Citation: Kelly KC, Evans K, Paun M, DeCoster MA (2016) Use of Randomized Submaximal Glutamate Stimulus to Interpret Glial Effects on Neuronal Calcium Dynamics. Neurochem Neuropharm Open Access 1: 107. doi:10.4172/2469-9780.1000107

Page 5 of 5

and extracellular residence time by decreasing glial cell content, one or more of different GLU receptor subtypes may be recruited [17] while cultures high in glia may on average reflect GLU stimulated activity at a more limited population of GLU receptors. Future studies will use pharmacological tools to test some of these hypotheses. For example, the NMDA receptor antagonist MK-801 would be expected to block or greatly inhibit glutamate-stimulated calcium influx at these threshold levels as we have previously shown for single stimuli [10]. Our current results may have some relevance to the idea of extrasynaptic GLU receptors, since diminished glia in our model may mimic the case where residence of extracellular GLU is elongated and thus "escapes" from the synapse leading to increased $\left[\mathrm{Ca}^{2+}\right]_{i}$, possibly to even excitotoxic levels [18]. Using recovery of baseline $\left[\mathrm{Ca}^{2+}\right]_{\mathrm{i}}$ (or lack thereof) of neurons in response to one GLU stimulus as a predictor of how cells will respond to a subsequent stimulus may have relevance to desensitization for information processing, potentially explaining waiting periods necessary for cells to handle one ensemble of stimuli before a second can be integrated. Berridge has described how the information processing capability of calcium can be enhanced by the availability of both amplitude and frequency modulation [19]. Our current results would indicate that the function (and density) of astrocytes and the order of stimulation could both be important filters for controlling this modulation in neurons. Furthermore, a recent report has described how glutamate-dependent neuroglial calcium signaling differs between young and adult brain [20]. Thus it is suggested that tripartite synapses consisting of pre- and postsynaptic neurons and surrounding glia may change with maturation and injury in the brain [21]. Since we used brain cell cultures derived from newborn rats in the studies described here, these recent findings in maturation differences would be consistent with our findings of highly oscillatory neuronal $\left[\mathrm{Ca}^{2+}\right]_{\mathrm{i}}$ responses to glutamate in cultures high in glial content. By depleting glia in our neuronal cultures, we have revealed the information processing capabilities of these neurons suggested by Grosche and Reichenbach [21]. In our case, it is revealed by showing that the order of submaximal stimulus with glutamate results in different outcomes when glia are largely absent, but not when they are there to homogenize the response.

\section{Acknowledgements}

This material is based upon work supported by the National Science Foundation under Grants No's. 1032176, 1116707 and 1463098. Any opinions, findings, and conclusions or recommendations expressed in this material are those of the authors and do not necessarily reflect the views of the National Science Foundation. Additional funding is gratefully acknowledged for the James $\mathrm{E}$. Wyche III and Walter Koss endowed Professorships from Louisiana Tech University to M.A.D. and K.E. respectively.

\section{References}

1. Huang R, Shuaib A, Hertz L (1993) Glutamate uptake and glutamate content in primary cultures of mouse astrocytes during anoxia, substrate deprivation and simulated ischemia under normothermic and hypothermic conditions. Brain
Res 618: 346-351.

2. DeCoster MA, Koenig ML, Hunter JC, Tortella FC (1992) Calcium dynamics in neurons treated with toxic and non-toxic concentrations of glutamate. Neuroreport 3: 773-776.

3. Bliss TV, Collingridge GL (1993) A synaptic model of memory: long-term potentiation in the hippocampus. Nature 361: 31-39.

4. Choi DW (1994) Calcium and excitotoxic neuronal injury. Ann NY Acad Sci 747 162-171.

5. Mattson MP (2007) Calcium and neurodegeneration. Aging Cell 6: 337-350.

6. Anderson CM, Swanson RA (2000) Astrocyte glutamate transport: review of properties, regulation, and physiological functions. Glia 32: 1-14.

7. Rothman SM, Olney JW (1995) Excitotoxicity and the NMDA receptor--still lethal after eight years. Trends Neurosci 18: 57-58.

8. Lyons SA, Chung WJ, Weaver AK, Ogunrinu T, Sontheimer H (2007) Autocrine glutamate signaling promotes glioma cell invasion. Cancer Res 67: 9463-9471.

9. Daniel B, DeCoster MA (2004) Quantification of SPLA2-induced early and late apoptosis changes in neuronal cell cultures using combined TUNEL and DAP staining. Brain Res Brain Res Protoc 13: 144-150.

10. DeCoster MA, Lambeau G, Lazdunski M, Bazan NG (2002) Secreted phospholipase A2 potentiates glutamate-induced calcium increase and cell death in primary neuronal cultures. J Neurosci Res 67: 634-645.

11. Cornell-Bell AH, Finkbeiner SM, Cooper MS, Smith SJ (1990) Glutamate induces calcium waves in cultured astrocytes: long-range glial signaling. Science 247: 470-473

12. Pizzo P, Burgo A, Pozzan T, Fasolato C (2001) Role of capacitative calcium entry on glutamate-induced calcium influx in type-I rat cortical astrocytes. neurochem 79: 98-109.

13. Xi Q, Tcheranova D, Basuroy S, Parfenova H, Jaggar JH, et al. (2011) Glutamateinduced calcium signals stimulate $\mathrm{CO}$ production in piglet astrocytes. Am J Physiol Heart Circ Physiol 301: H428-433.

14. Rosenberg PA, Aizenman E (1989) Hundred-fold increase in neurona vulnerability to glutamate toxicity in astrocyte-poor cultures of rat cerebral cortex. Neurosci Lett 103: 162-168.

15. Swanson RA (1992) Astrocyte glutamate uptake during chemical hypoxia in vitro. Neurosci Lett 147: 143-146.

16. Bacci A, Verderio C, Pravettoni E, Matteoli M (1999) The role of glial cells in synaptic function. Philos Trans R Soc Lon B Biol Sci 354: 403-409.

17. Nakanishi S (1992) Molecular diversity of glutamate receptors and implications for brain function. Science 258: 597-603.

18. Hardingham GE, Fukunaga Y, Bading $\mathrm{H}$ (2002) Extrasynaptic NMDARs oppose synaptic NMDARs by triggering CREB shut-off and cell death pathways. Nat Neurosci 5: 405-414.

19. Berridge MJ (1997) The AM and FM of calcium signalling. Nature 386: 759-760.

20. Sun W, McConnell E, Pare JF, Xu Q, Chen M, et al. (2013) Glutamatedependent neuroglial calcium signaling differs between young and adult brain Science 339: 197-200

21. Grosche A, Reichenbach A (2013) Neuroscience. Developmental refining of neuroglial signaling. Science 339: 152-153. 